\title{
La transmission des patrimoines paysans : clé de voûte de la reproduction sociale et faux problème
}

L'exemple du canton de Plélan-le-Grand au milieu du xix siècle

\section{Fabrice Boudjaaba}

\section{(2) OpenEdition \\ 1 Journals}

\section{Édition électronique}

URL : http://journals.openedition.org/abpo/114

DOI : 10.4000/abpo. 114

ISBN : 978-2-7535-1514-7

ISSN : 2108-6443

\section{Éditeur}

Presses universitaires de Rennes

\section{Édition imprimée}

Date de publication : 30 juin 2009

Pagination : 141-163

ISBN : 978-2-7535-0941-2

ISSN : 0399-0826

\section{Référence électronique}

Fabrice Boudjaaba, «La transmission des patrimoines paysans : clé de voûte de la reproduction sociale et faux problème ", Annales de Bretagne et des Pays de l'Ouest [En ligne], 116-2 I 2009, mis en ligne le 30 juin 2011, consulté le 01 mai 2019. URL : http://journals.openedition.org/abpo/114 ; DOI : $10.4000 / a b p o .114$ 


\title{
La transmission des patrimoines paysans : clé de voûte de la reproduction sociale et faux problème

\author{
L'exemple du canton de Plélan-le-Grand
} au milieu du XIX ${ }^{\mathrm{e}}$ siècle
}

\author{
Fabrice BOUDJAABA \\ Chargé de recherche au CNRS \\ CERHIO - université Rennes 2 Haute-Bretagne
}

\begin{abstract}
La question de transmission des exploitations paysannes préoccupe au premier chef les historiens ruralistes, en particulier ceux de l'époque moderne ${ }^{1}$ et de la France du xix ${ }^{\mathrm{e}}$ siècle, et, au-delà, les spécialistes des systèmes de parenté et des pratiques successorales ${ }^{2}$. Dans un contexte où la rente foncière reste le fondement des fortunes et le travail de la terre l'activité principale d'une nette majorité des populations, l'intérêt porté à cette question se comprend aisément.
\end{abstract}

Dans ce cadre économique, les possibilités d'asseoir sa reproduction familiale sur autre chose que la propriété de la terre, ou son exploitation, sont relativement réduites. En effet, la reproduction sociale et professionnelle au sein des familles est compliquée par le fait que la quantité de fortune foncière et de travail que procure la terre n'est pas extensible à l'infini. En d'autres termes, au moment de la succession, l'exploitation agricole, ou tout au moins le patrimoine foncier, est menacée de dislocation entre les différents héritiers qui, chacun, réclament un droit légitime sur ce bien;

1. À titre d'exemple le guide d'histoire agraire publié en 1999 par l'Association d'Histoire Rurale comprend 45 titres consacrés au thème "Famille, patrimoine et transmission " pour l'Ancien Régime; MoRICEAU, Jean-Marc, La terre et les paysans aux XVIT et XVIII siècles. Guide d'histoire agraire, Caen, Bibliothèque d'histoire rurale, 1999.

2. À commencer par les nombreux travaux d'historiens mais également d'ethnologues sur les systèmes familiaux pyrénéens; GoY, Joseph et CHIVA, Isaac (dir.), Les Baronnies des Pyrénées, Paris, EHESS, 1981 et 1986. Voir également la mise au point historiographique dans BARTHÉLÉMY, Thiphaine, "Les modes de transmission du patrimoine. Synthèse des travaux effectués depuis quinze ans par les ethnologues de la France ", Études Rurales, p. 110 à 112, 1988, p. 195-212. 
de ce fait elle risque de se transformer en deux, trois ou quatre petites exploitations non viables, faute d'atteindre une taille critique minimale. Contrairement à notre société actuelle où la survie de nombre d'exploitations agricoles est menacée par la pénurie d'héritiers volontaires pour reprendre le métier des parents, parce qu'ils disposent d'un éventail beaucoup plus large de possibilités professionnelles hors du secteur agricole, le problème serait donc plutôt $a$ priori, pour les sociétés traditionnelles et jusqu'au moins le milieu du XIX ${ }^{\mathrm{e}}$ siècle ${ }^{3}$, la pléthore de successeurs potentiels. Faute de véritables débouchés professionnels véritablement attrayants, sauf à considérer le travail industriel comme une perspective enviable à cette époque, les historiens ont en général considéré que la transmission de l'exploitation agricole constituait le problème majeur pour les familles et la reproduction sociale de leurs enfants.

C'est à la mesure exacte de la réalité du problème de la transmission et du morcellement des patrimoines qui s'ensuit que nous souhaiterions nous attacher dans cet article, à partir des successions déclarées dans le canton de Plélan en 1856. Nous aimerions aussi comprendre dans quelle mesure les paysans cherchaient ou non à contourner ce problème par des choix économiques spécifiques ou une adaptation de leur comportement familial et démographique, et notamment une transformation de leur fécondité.

\section{La transmission des exploitations}

et leur morcellement au $\mathrm{XIX}^{\mathrm{e}}$ siècle : position du problème

La question du morcellement explique en partie l'intérêt des historiens pour les systèmes successoraux de la France d'Ancien Régime et les dispositions successorales du Code civil. Les chercheurs interrogeant un raisonnement de type malthusien sur les rapports entre la quantité d'hommes et la quantité de richesses, en l'occurrence de terres, disponibles, ont tenté de comprendre par quels moyens les familles parvenaient à assurer l'avenir de leurs enfants et celui de leur exploitation. C'est pourquoi les systèmes inégalitaires ont tant passionné et continuent ${ }^{4}$ de passionner les historiens : ils offrent une solution a priori simple au maintien des exploitations d'une génération à l'autre par la désignation d'un héritier et l'exclusion des autres contraints au célibat et/ou à la mobilité géographique et/ou professionnelle.

Le Code civil, dans ce contexte, est apparu comme une rupture dans les pratiques successorales et les processus de reproduction familiale, même si on oublie trop souvent que ce texte ne fait que reprendre pour l'essentiel

3. " Imaginer les campagnes françaises dépeuplées était une pure vie de l'esprit " puisque la population rurale française atteint son maximum en 1846 selon Jean-Pierre POUSSOU et al. dans DuPÂQUIER, Jacques (dir.), Histoire de la population française, Paris, PUF, 1995, p. 180.

4. La question de la transmission reste centrale dans l'ouvrage récent de SABEAN, David, Teuscher, Simon et Mathieu, Jon (éd.), Kinship in Europe. Approaches to Long-term Developments (1300-1900), Oxford/New York, Berghahn Books, 2007. 
un système d'héritage ancien, celui de la coutume de Paris, strictement égalitaire, quel que soit le sexe des héritiers. Ce nouveau droit a d'ailleurs suscité un certain nombre d'oppositions se manifestant par la perpétuation d'avantages au bénéfice d'un des enfants dans les régions où l'ancienne loi prévoyait la désignation d'un héritier unique ${ }^{5}$.

Pourtant, il semble que beaucoup de travaux historiques ${ }^{6}$, et $a$ fortiori ethnologiques ${ }^{7}$, ont sans doute (exagérément) focalisé sur le problème du partage des successions et la division des patrimoines. Cette attention soutenue relève, nous semble-t-il, pour partie de problèmes de sources et de méthodes. L'étude du devenir d'une exploitation sur plusieurs générations est en effet un travail souvent très complexe à réaliser. Paradoxalement le moment de la succession, a priori si important pour les exploitations et les familles, compte parmi les transferts de propriété les plus mal enregistrés. La monarchie française n'est jamais parvenue à contraindre ses sujets à déclarer la succession des défunts ${ }^{8}$. C'est seulement au XIX ${ }^{\mathrm{e}}$ siècle que cet enregistrement s'impose à la suite de la loi de germinal an VII. Cependant, même dans ce cas, une déclaration de succession ne décrit pas la manière dont le patrimoine est ensuite redistribué entre les différents héritiers. Les actes de partage ne sont pas systématiquement enregistrés ni même notariés. On sait en outre que le processus de transfert peut commencer bien avant le décès des parents par des donations ou des avances d'hoirie notamment lors du mariage des enfants ${ }^{9}$. Suivre le destin de tous les enfants d'un même couple est également une opération très complexe. Or, si on ne connaît pas le destin de tous les enfants il est évidemment difficile d'expliquer pourquoi et comment tel enfant succède à ses parents plutôt qu'un autre. Ces difficultés ont conduit à privilégier l'étude de cas, c'est-à-dire d'exploitations bien identifiables ou de quelques familles dont la généalogie est bien établie et qui laissent de bonnes archives. Cela a pu contribuer à notre sens à exagérer l'importance de la question de la succession dans les processus de reproduction et de mobilité sociale dans les campagnes traditionnelles en négligeant une approche plus globale, macro si on peut écrire, de ces problèmes.

5. C'est l'une des raisons d'une certaine défiance des paysans vis-vis de la Révolution. Voir Goy, Joseph, «Transmission successorale et paysannerie pendant la Révolution française : un grand malentendu ", Études rurales, avril-décembre 1988, p. 45-56.

6. C'est le constat que fait Gérard BÉAUR dans " Trop de stratégie? Transmission, démographie et migration dans la Normandie rurale du début du XIX ${ }^{\mathrm{e}}$ siècle (Bayeux, Domfront, Douvres, Livarot) " dans Histoire des familles de la démographie et des comportements. Mélanges en l'honneur de Jean-Pierre Bardet, Paris, PUPS, 2007, p. 37-53.

7. Récemment encore dans PINGAUD, Marie-Claude, Faire ses partages. Terres et parentèles dans le Perche $\mathrm{XIX}^{e}-\mathrm{XX}$ e siècles, Rennes, PUR, 2006.

8. Du moins l'enregistrement des successions en ligne directe, seules les successions collatérales faisant au $\mathrm{XVIII}^{\mathrm{e}}$ siècle l'objet d'une déclaration consignée dans les registres du centième denier.

9. Sur ce point, voir DÉROUET, Bernard, « Dot et héritage : les enjeux de la chronologie de la transmission " dans L'Histoire grande ouverte. Hommages à Emmanuel Le Roy Ladurie, Paris, Fayard, 1997, p. 284-296. 
G. Béaur, dans un article ${ }^{10}$ essentiellement consacré à la question des migrations et de leur rapport avec la question des stratégies familiales, a ainsi signalé pour la région de Bayeux que le problème de la dispersion des patrimoines fonciers entre les héritiers était moins épineux qu'on pouvait le penser a priori dans la mesure où le nombre de défunts qui n'ont pas d'héritiers directs était très important et que la moyenne du nombre d'enfants des défunts était seulement de 1,66 enfant. Dans ces conditions, beaucoup de successions se réglaient simplement. Le combat entre héritiers pour la reproduction sociale à l'identique, dans les pas et à la place du père, n'avait souvent pas lieu faute de combattants. Pour la région de Vernon ${ }^{11}$, quoique dans une perspective légèrement différente, nous étions arrivé à un constat de même nature. Dans ces conditions, le problème du maintien des exploitations et de leur devenir se pose avec moins d'acuité qu'il n'y paraît dans la mesure où une grande partie des successions n'aboutissent pas à un éparpillement des patrimoines entre de multiples ayants droit ou à un éparpillement géographique des héritiers contraints de partir vivre ailleurs d'autre chose que de ces poussières de patrimoine.

Cependant, quelle que soit la pertinence de ces deux échantillons, celui de Bayeux dans les années 1870 ou celui de Vernon dans les années 18101820 , il faut signaler qu'il s'agit de deux régions où la fécondité des femmes a chuté très précocement. La région de Vernon a été identifiée comme l'un des premiers bassins ruraux où le monde paysan imite les pratiques de limitation des naissances déjà fort répandues dans les villes de la fin de l'Ancien Régime ${ }^{12}$. La Normandie dans son ensemble dans le dernier tiers du $\mathrm{XIX}^{\mathrm{e}}$ siècle a déjà depuis longtemps intégré ce type de comportement. Dans ces deux échantillons le problème de la transmission des exploitations et de l'intégrité des patrimoines trouve donc très largement sa solution dans une transition démographique déjà bien entamée aux deux périodes d'analyse.

Il ne s'agit pas pour autant de nier l'intérêt de cette approche globale du rapport entre transmission des patrimoines et démographie. Bien au contraire, cette démarche a le mérite de relativiser certaines conclusions tirées d'études de cas, par définition, plus souvent centrées sur des transmissions à problème que sur celles qui se "passent bien ". Cependant, nous souhaiterions ici approfondir la question des rapports entre patrimoines, transmission et descendance pour un territoire où la démographie pose $a$ priori un problème plus vif qu'en Normandie. Bref, nous voudrions

10. BÉAUR, Gérard, « Mobiles ou sédentaires? Les familles rurales normandes face au problème de la migration au XIX ${ }^{\mathrm{e}}$ siècle (Bayeux, 1871-1874) " dans Luigi LORENZETTI, Anne-Lise HEAD-KÖNIG et Joseph GoY (éd.), Marchés migrations et logiques familiales dans les espaces français, canadien et suisse, 18e-20e siècles, Bern, Peter Lang, 2005.

11. BoudJAABA, Fabrice, Des paysans attachés à la terre? Familles, marchés et patrimoines dans la région de Vernon (1750-1830), Paris, PUPS, 2008.

12. BARDET, Jean-Pierre, Rouen au XVII et au XVII 'e siècle. Les mutations d'un espace social, Paris, SEDES, 1983, 2 vol. 
poser cette question pour un territoire où les comportements malthusiens ne l'emportent pas encore au milieu du XIX ${ }^{\mathrm{e}}$ siècle : la Bretagne, et plus précisément, le canton de Plélan en Ille-et-Vilaine. L'expression de comportements malthusiens étant utilisée ici dans un sens très large : elle désigne simplement une réduction sensible de la descendance par une limitation des naissances sans que ce comportement soit nécessairement associé chez les individus concernés à une réflexion " consciente " sur la production de richesse, c'est-à-dire, au plan individuel, à une stratégie de protection du patrimoine familial contre les risques de morcellement que lui font courir le Code civil lorsque la descendance d'un individu est nombreuse ${ }^{13}$.

À l'inverse de la Normandie, la Bretagne a en effet conservé une fécondité plus élevée que la moyenne nationale durant tout le XIX ${ }^{\mathrm{e}}$ siècle $^{14}$. Il n'est pas question ici pour nous de discuter des raisons de ce comportement (moindre déchristianisation, moindre influence urbaine...) mais seulement dans un premier temps de mesurer l'intensité des problèmes de transmission successorale pour cette économie rurale et d'analyser les rapports entre la taille des patrimoines à transmettre et la taille des familles. Dans un second temps, quittant ce niveau global d'analyse pour prendre en compte les individus, il s'agira de comprendre si les comportements démographiques peuvent s'expliquer au moins en partie par des motivations patrimoniales. Une descendance réduite est-elle associée à une plus grande valeur des patrimoines? Dans ce cas, faut-il lire cette corrélation comme le signe d'une volonté de transmettre déterminante des comportements démographiques? Cette question est évidemment à mettre en relation avec le débat sur le malthusianisme de la paysannerie française au XIX $x^{\mathrm{e}}$ siècle selon lequel l'attachement présumé à la conservation et à la transmission

13. Il serait évidemment très intéressant de poser dans les mêmes termes le problème de la transmission pour la France rurale d'Ancien Régime, c'est-à-dire pour une société qui ne connaît pas, ou encore très peu, la limitation des naissances et dont l'économie est clairement préindustrielle. Malheureusement il est impossible faute de sources pour cette période de connaître l'ensemble des successions. Dans ces conditions la Bretagne du XIX ${ }^{\mathrm{e}}$ siècle constitue un observatoire privilégié par ses pratiques démographiques " anciennes " et une économie en retard de modernisation.

14. Selon Noël Bonneuil l'indice $\mathrm{I}_{f}$ de Coale (qui mesure la fécondité d'une population par comparaison avec la fécondité " maximum " des Huttérites) du département de l'Illeet-Vilaine s'élève encore en 1856 à 0,313 alors qu'il est, en moyenne nationale, de 0,307. Mais surtout dans les décennies précédentes (qui nous intéressent davantage car le nombre d'héritiers en 1856 en dépend largement) cet indice était encore bien plus élevé (0,510 en 1806 contre une moyenne nationale de 0,393). Le canton de Plélan a sans doute un indice encore plus élevé que la moyenne départementale dans la mesure où il s'agit d'un canton très rural et surtout limitrophe du Morbihan où la fécondité est encore plus élevée (indice de Coale de 0,641 en 1806, 0,424 en 1856). La fécondité bretonne au XIX ${ }^{\mathrm{e}}$ siècle est donc très différente de celle de la Normandie, qu'il s'agisse de l'Eure ou du Calvados qui sont les deux départements où elle est la plus faible dès le début du XIX ${ }^{\mathrm{e}}$ siècle $(0,246$ en 1806 et 0,217 en 1856 pour le Calvados; 0,306 en 1806 et 0,215 en 1856 pour l'Eure). Bonneull, Noël Transformation of the French Demographic Landscape, Oxford, Clarendon Press, 1997. 
de son patrimoine, expliquerait le comportement démographique du petit paysan propriétaire ${ }^{15}$.

\section{Le canton de Plélan au milieu du XIX ${ }^{\mathrm{e}}$ siècle}

Le choix du canton de Plélan-le-Grand s'est imposé simplement. La région au milieu du XIX ${ }^{\mathrm{e}}$ siècle est encore très largement rurale. Bien qu'appartenant à l'llle-et-Vilaine, département breton ${ }^{16}$ le plus ouvert aux évolutions économiques du siècle avec la Loire-inférieure ${ }^{17}$, le canton se situe à son extrémité ouest, à la limite du Morbihan; il ne dispose d'aucune activité industrielle remarquable en dehors des anciennes forges de Paimpont; il est suffisamment éloigné des villes susceptibles de l'intégrer à leur aire d'influence immédiate; Rennes est à plus de 35 kilomètres. Il s'agit donc d'une région dont les structures économiques reposent encore très largement sur l'agriculture. Les forges, dont l'existence remonte à la fin du Xvil siècle, faute de développement nouveau et d'une modernisation, restent une source d'activité très secondaire qui commence à décliner au milieu du $\mathrm{XIX}^{\mathrm{e}}$ siècle ${ }^{18}$. Dans ces conditions, la question de la transmission des patrimoines fonciers et des exploitations agricoles se pose à Plélan avec toute son acuité dans la mesure où le marché du travail local ne fournit pas aux enfants de paysans d'alternative suffisante à l'activité agricole. En dehors de la migration dont les proportions sont encore limitées ${ }^{19}$, la solution passe pour l'essentiel d'entre eux par le travail de la terre sur place ${ }^{20}$.

Nous disposons pour le $\mathrm{xIX}^{\mathrm{e}}$ siècle d'une source extrêmement commode pour appréhender la fortune des individus : les registres de mutation par décès. Depuis la loi de germinal an VII ${ }^{21}$, chaque décès doit donner lieu à

15. Ce débat est à la fois alimenté par une réalité démographique, la précocité de la chute de la fécondité en France par rapport aux autres pays européens, et par l'opinion d'un certain nombre de penseurs libéraux du XIX ${ }^{\mathrm{e}}$ siècle favorables à une limitation des naissances dans les milieux populaires. Rappelons l'invite de Jean-Baptiste Say " aux hommes à faire des épargnes plutôt que des enfants " cité dans DuPÂQUIER, Jacques (dir.), Histoire de la population..., op. cit., p. 369.

16. Bien entendu le cadre départemental n'est pas, en particulier au XIX ${ }^{\mathrm{e}}$ siècle, nécessairement le plus pertinent pour délimiter des espaces économiques homogènes mais nous l'utilisons tout de même car les statistiques démographiques à notre disposition s'inscrivent dans ce cadre de référence.

17. BouRRIGAUD, René, Le Développement agraire en Loire-Inférieure au XIXe siècle. Essai sur l'histoire des techniques et des institutions, Thèse de l'université de Nantes, 1993.

18. Les Forges de Paimpont. Une activité industrielle du XVIf au XIX siècle, étude publiée par la fédération Carrefour de Trécélien, imprimerie de Chatenay, sans date.

19. Dans le canton, sur 504 héritiers en ligne directe répertoriés dans le registre de mutation par décès de 1856 , seuls 44 , soit $8,7 \%$, ont migré hors du canton au moment où leur parent est décédé.

20. À cela s'ajoute le travail fourni par l'exploitation de la forêt de Paimpont qui se manifeste dans les sources notamment par la présence de quelques sabotiers. Mais l'absence de bûcherons dans nos sources indique que, déjà en 1856, les forges de Paimpont ne fournissent plus beaucoup de travail aux habitants.

21. Pour connaître le détail sur la mise en place de cette loi voir DAUMARD, Adeline, Les Fortunes françaises au XIX $X^{e}$ siècle, Paris, Mouton, 1973, p. 4 sqq. 
une déclaration devant la régie de l'Enregistrement et des domaines dans laquelle l'un des héritiers vient déclarer, en son nom propre et au nom des cohéritiers, le contenu mobilier et immobilier ainsi que la valeur du patrimoine du défunt situé dans la circonscription du bureau. Les biens possédés dans d'autres bureaux ne sont donc pas déclarés. Théoriquement, même les successions nulles, celles des enfants notamment, doivent être déclarées, ce qui est loin d'être toujours le cas. En revanche, pour les successions non nulles, la source se révèle d'une très grande fiabilité. Les maires des communes doivent en effet rapporter à l'administration fiscale la liste des défunts de leur village, ce qui permet aux employés de l'Enregistrement de vérifier qu'une déclaration de succession a bien été effectuée dans les six mois qui suivent le décès d'un individu. Cette source, d'une grande qualité, a finalement été peu utilisée depuis les travaux initiaux de A. Daumard sur les fortunes parisiennes. Il faut signaler cependant que dans le cadre du volet patrimonial de la grande enquête des 3000 familles TRA initiée par J. Dupâquier cette source a été abondamment utilisée mais tous les résultats de ces dépouillements, encore inachevés, sont loin d'avoir été exploités ${ }^{22}$. L'enquête menée actuellement sur Plélan n'a évidemment pas cette ampleur puisqu'elle repose sur le dépouillement de trois années (1836, 1856 et 1876). Dans cet article nous nous concentrerons sur l'année $1856^{23}$ dont les données ont été intégralement dépouillées et traitées. Pour l'année 1856 le registre de succession contient 221 déclarations. L'effectif est relativement modeste car les dépouillements restent longs, une même déclaration peut en effet s'étendre sur plusieurs pages de ces grands registres. Il est cependant suffisant pour proposer une approche chiffrée de la transmission des patrimoines et de leur dispersion.

L'échantillon est composé presque exclusivement de ruraux et principalement de paysans propriétaires. Le registre indique la profession de 181 des 221 défunts. Parmi eux 60 \% sont déclarés comme cultivateur ou laboureur. Un seul défunt est " journalier». Sans doute faut-il ajouter à ce monde paysan la plupart des 40 individus dont la profession n'est pas indiquée (dont 19 sont des femmes) et une partie des neuf "soldats " comptabilisés. Si on exclut treize défunts signalés comme " mineurs " en lieu et place de la profession dans le registre, il paraît évident que la source reflète essentiellement le niveau de fortune au décès d'un monde de petits et de moyens cultivateurs. Nous avons en effet comptabilisé seulement deux charpentiers, un cloutier, trois tisserands etc., au total 17 artisans, ruraux pour l'essentiel eux aussi. La bourgeoisie et le monde urbain sont absents de la source, à l'exception de la duchesse de Narbonne-Pelet, décédée à

22. Bourdieu, Jérôme, Postel-Vinay, Gilles et SuwA-EISEnMann, Akiko, " Défense et illustration de l'enquête des 3000 familles. L'exemple de son volet patrimonial ", Annales de démographie historique, 2004-1, p. 19-52, ainsi que dans le même volume ARRONDEL, Luc et Grange, Cyril, "Successions et héritiers dans la société rurale du XIX ${ }^{\mathrm{e}}$ siècle : l'exemple des familles TRA de Loire-inférieure ", p. 53-77.

23. Archives départementales d'Ille-et-Vilaine, 3Q 27333. 
Versailles, qui laisse, par testament, plus de 60000 francs de biens dans le canton à Armand Étienne de Charrette.

\section{Transmission et intégrité du patrimoine : la mesure globale du problème à Plélan}

Dans quelle proportion la transmission des patrimoines se traduit-elle par un risque véritable de dispersion de la fortune accumulée par la génération précédente et, dans le cas des paysans, par un risque de morcellement de l'exploitation?

\section{Descendance "définitive » et transmission des patrimoines}

Une première approche de la dispersion des patrimoines par la transmission consiste tout simplement à compter le nombre d'héritiers en ligne directe de chaque succession. Plus de $39 \%$ des successions n'ont pas d'héritiers en ligne directe en 1856 à Plélan. 87 des 221 successions ouvertes durant cette année sont en fait des successions " non directes " c'est-à-dire en ligne collatérale ( 80 cas), " entre époux " strictement ${ }^{24}$ (5 cas) ou à des étrangers ( 2 cas). Près de quatre successions sur dix n'ont donc pas d'effet immédiat sur la reproduction sociale et le maintien des exploitations familiales dans la mesure où, s'agissant d'individus sans descendance, la question n'a pas de sens. Même si leur patrimoine est divisé à partir du moment où ils ont plusieurs héritiers, cet héritage reste un bénéfice collatéral et ne constitue pas le cœur du processus de reproduction familiale des parents aux enfants. Si on ajoute à ce groupe les successions en ligne directe à un seul héritier (17 cas) et qui donc ne posent pas de problème de morcellement, on constate alors que $47 \%$ des successions sont étrangères au problème de la reproduction familiale à l'identique.

Ce chiffre qui vient relativiser fortement le problème de la transmission des patrimoines, et particulièrement des exploitations agricoles, n'est-il qu'un artefact induit par l'amalgame qui est opéré entre successions directes et successions " non directes " sous toutes leurs formes? Ce serait notamment le cas si la grande majorité des successions collatérales concernaient de jeunes enfants. Légalement tout défunt fait l'objet d'une déclaration de succession même s'il ne possède rien. Mais, à Plélan, comme dans beaucoup d'autres bureaux d'enregistrement, les enfants décédés ne font l'objet d'aucune déclaration de succession. Dans ces conditions ces défunts adultes font donc partie de la descendance "définitive " de leurs parents et sont à prendre en compte dans les processus de reproduction

24. Ce chiffre ne tient pas compte de toutes les successions "collatérales et entre époux " ou " directe et entre époux " car dans ces cas à successeurs " mixtes ", le véritable successeur n'est pas l'époux qui bénéficie seulement d'un usufruit sur une partie des biens. Cependant, dans les cinq cas en question à la suite d'un testament, le conjoint est héritier en pleine propriété des biens du défunt. Il s'agit donc d'une forme de succession non directe au même titre que la succession collatérale. 
sociale et familiale au même titre que ceux qui ont des enfants. Pourtant la proportion de successions de type collatéral semble particulièrement élevée à Plélan quand on la compare aux régions pour lesquelles nous disposons de données.

En étudiant les registres de mutation par décès de la région de Vernon, en Normandie, entre 1809 et 1824 nous avions en effet constaté que la part des successions collatérales était seulement de $19 \%$. Dans la région de Bayeux (ville exclue), à une époque plus tardive, G. Béaur ${ }^{25}$ observait une proportion de successions collatérales intermédiaire entre la situation bretonne et celle de Vernon puisque, entre 1871 et 1874, $30 \%$ des défunts n'avaient pas d'enfant survivant. Pourtant, dans ces deux cas, la question de la transmission ne se pose pas en des termes plus complexes qu'à Plélan. Si les successions collatérales y sont moins nombreuses, les successions directes simples, c'est-à-dire à un seul héritier y sont en revanche plus nombreuses. À Vernon, en effet $32 \%$ des successions sont des successions directes à un héritier, ce qui est le cas " d'environ $30 \%{ }^{26}$ " des successions dans la région de Bayeux. Dans ces conditions, $51 \%$ des successions à Vernon dans les années 1810-1820 et près de $60 \%$ des successions de la région de Bayeux en 1871-1874 ne posent pas de problème en termes de maintien des exploitations et d'intégrité des patrimoines. Au regard de ces deux exemples la situation paraît donc plus compliquée à Plélan alors que 47 \% des successions sont pourtant sans conséquence sur l'intégrité des patrimoines. Mais globalement on peut, quel que soit l'échantillon, retenir qu'une moitié des successions ne produit pas directement de morcellement des patrimoines fonciers.

$\mathrm{Si}$, au XIX ${ }^{\mathrm{e}}$ siècle, les deux régions ont en commun un système de partage des successions égalitaire imposé par le Code civil mais également des traditions successorales de type égalitaire, avec un bémol à ce principe concernant les filles dans la Normandie d'Ancien Régime ${ }^{27}$, la Bretagne et la Normandie n'empruntent pas les mêmes chemins pour aboutir au même résultat. La simplification du processus de transmission passe pour Plélan par un fort taux de défunts sans descendance tandis que, pour la Normandie, cela passe, tout autant dans le cas de Bayeux, et avant tout dans le cas de Vernon, par une plus forte proportion de défunts à enfant unique. On peut donc émettre l'hypothèse que le système de transmission patrimoniale et de reproduction sociale trouve sa régulation en Bretagne dans un contexte régional de fécondité encore élevée au XIX ${ }^{\mathrm{e}}$ siècle par un plus fort taux de célibat $^{28}$. En Normandie, en revanche, le célibat définitif est moindre mais la

\footnotetext{
25. BEAUR, Gérard, " Trop de stratégie?... », op. cit., p. 267.

26. Ibidem.

27. BOUDJAABA, Fabrice, «Femmes, patrimoine et marché foncier dans la région de Vernon (1760-1830) : le patrilignage normand face au Code civil ", Histoire et sociétés rurales, 28, 2007-2, p. 33-66.

28. Les données fournies par l'Histoire de la population française indiquent que la Bretagne, quel que soit le département considéré, présente toujours des taux de célibat définitif parmi les plus élevés de France. Pour la génération des Bretonnes de 1799-1803 (dont un certain
} 
plus forte limitation volontaire des naissances, identifiée dès le xvIII ${ }^{e}$ siècle dans cette région ${ }^{29}$ accroît le nombre de défunts à la descendance réduite et en particulier le nombre de familles à un ou deux enfants survivants.

Le tableau 1 confirme la distribution très différente de la descendance finale des défunts entre Plélan et la région de Vernon. Non seulement la part de défunts sans descendance est bien plus importante en Bretagne que dans notre échantillon normand mais les enfants, quand le défunt en a, sont en revanche bien plus nombreux à Plélan; 58 \% des défunts qui ont une descendance ont un ou deux enfants seulement à Vernon alors qu'ils sont seulement $31 \%$ dans ce cas à Plélan. À l'inverse le nombre de défunts à famille nombreuse (quatre enfants survivants au moins) est bien plus grand à Plélan : $51 \%$ des défunts qui ont des descendants directs ont au moins quatre enfants, contre seulement $18 \%$ à Vernon ${ }^{30}$. G. Béaur ne fournit pas ce type de données dans son article mais indique que dans la région de Bayeux seulement $6 \%$ des défunts laissent plus de quatre enfants ${ }^{31}$.

Tableau 1 - Nombre d'héritiers en ligne directe par succession à Vernon et à Plélan

\begin{tabular}{|l|c|c|}
\hline \multirow{2}{*}{$\begin{array}{l}\text { Nombre de descendants } \\
\text { en ligne directe }\end{array}$} & \multicolumn{2}{|c|}{ Nombre de successions en pourcentage } \\
\cline { 2 - 3 } Sans descendance & 19,0 & Plélan 1856 \\
\hline 1 & 32,0 & 39,4 \\
\hline 2 & 15,1 & 7,7 \\
\hline 3 & 10,9 & 11,3 \\
\hline 4 & 8,1 & 10,4 \\
\hline 5 & 6,2 & 12,2 \\
\hline 6 & 1,2 & 7,2 \\
\hline 7 & 2,1 & 6,8 \\
\hline 8 & 0 & 2,2 \\
\hline Plus de 8 & 0 & 1,8 \\
\hline "Plusieurs & 5,2 & 0,9 \\
\hline Effectif & 515 & 0 \\
\hline
\end{tabular}

a. BOUDJAABA, Fabrice, Des paysans attachés à la terre..., op. cit., tableau VI-7, p. 228.

Les registres de successions reflètent donc des comportements familiaux et contraceptifs très différents selon la région considérée. La Bretagne

nombre des individus décédés en 1856 sont issus), le taux de célibat définitif est compris entre 10 et $15 \%$ en Ille-et-Vilaine et est supérieur à $20 \%$ pour le Morbihan limitrophe du canton de Plélan. DuPÂQUIER, Jacques, Histoire de la population..., op. cit., p. 426.

29. BARDET, Jean-Pierre (dir.), Histoire des populations de l'Europe, Paris, Fayard, t. 2, p. 314.

30. En incluant les défunts à "plusieurs " héritiers. L'imprécision vient du fait que les successions ont été relevées dans les tables de successions et non dans les registres de successions et qu'elles sont moins précises sur ce point.

31. BÉAUR, Gérard, "Trop de stratégie?... ", op. cit., p. 267. 
conserve une fécondité forte au milieu du XIX ${ }^{\mathrm{e}}$ siècle qu'elle semble compenser partiellement en quelque sorte sur le plan patrimonial par un plus fort taux de célibat définitif, quand la Normandie a déjà fortement limité ses naissances dans les années 1810. G. Béaur compte, rappelons-le, 1,66 enfant par succession; à Plélan ce chiffre s'élève à 2,25 . À Vernon il n'est pas calculable précisément faute de disposer systématiquement du nombre exact d'enfants pour chaque succession.

\section{L'apport des successions collatérales à la transmission intergénérationnelle des patrimoines}

Toutefois, tous les décès sans descendance enregistrés à Plélan peuvent-ils vraiment être considérés comme des formes de régulation du système de transmission patrimoniale? Si on s'en tient à une approche globale la réponse est positive : à la génération 1 environ quatre personnes sur dix n'ont pas d'enfant. Pour les six autres le problème de la division du patrimoine entre les enfants se pose évidemment dès lors qu'ils ont plus de deux enfants mais l'apport en ligne collatérale des quatre individus de la génération 1 (frères et sœurs), morts sans descendance, vient compenser cette dilution du patrimoine à la génération 2 . Les trois couples de la génération 1, c'est-à-dire les six individus disposant d'une descendance à la génération 1, peuvent en quelque sorte s'autoriser à avoir jusqu'à dix enfants au total soit 3,33 enfants par couple, soit bien plus que les 2,25 enfants qu'ils ont en réalité.

Cette approche globale ne permet cependant pas de rendre compte de la manière dont se déroulent ces processus de transmission dans les familles pour au moins deux raisons. D'une part, la totalité des patrimoines ne circule pas par la voie successorale. Tous les biens vendus par les individus de la génération 1 notamment en vue de financer leurs vieux jours n'apparaissent pas dans les registres de mutation par décès mais ont emprunté les voies du marché pour changer de mains ${ }^{32}$. En effet en l'absence de système de financement collectif de la vieillesse, les individus peuvent être conduits à vendre une partie de leurs terres pour vivre quand ils n'ont plus les moyens physiques de les exploiter. Ce schéma général, commun à nombre de régions où les ménages ont une dominante nucléaire, peut s'appliquer à la Bretagne même si on y rencontre, il est vrai, plus fréquemment qu'en Normandie ou en Île de France par exemple, des formes de ménages élargis aux grandsparents ${ }^{33}$. Toutefois la Bretagne n'est pas un pays de système à maison où la conservation de l'exploitation implique des structures familiales de type complexe comme dans les Pyrénées par exemple. Même si la Bretagne compense fortement les contraintes du partage égalitaire par un taux plus prononcé de célibat définitif la circulation marchande des biens-fonds en elle-même contribue à relativiser les effets du processus de transmission sur

32. BOUDJAABA, F., op. cit., 2008, p. 189-194.

33. Segalen, Martine, Quinze générations de Bas-Bretons, Paris, PUF, 1985. 
le morcellement des héritages et nous rappelle que le processus de reproduction sociale ne repose jamais exclusivement sur l'héritage foncier, même dans une économie où la rente foncière reste dominante.

D'autre part, il n'est pas certain que ceux qui meurent sans descendance soient des individus qui pèsent autant que les autres dans les processus de transmission patrimoniale d'une génération à l'autre. En d'autres termes, il faut se demander si le patrimoine des successions collatérales est d'une valeur comparable à celui des successions en ligne directe ou si il n'est pas plus réduit? Une personne sans descendance n'a peut-être pas le même type de parcours patrimonial qu'un individu marié avec enfants parce qu'il n'a pas de motif évident de transmission dans la mesure où il ne cherche pas à préparer l'avenir de sa descendance. Si cet individu est célibataire on peut également supposer qu'il s'agit parfois d'un individu mort plus prématurément, en tout cas plus jeune que la plupart des défunts avec enfants et que, par conséquent, il possède potentiellement moins de biens-fonds que ce dernier.

Pour comprendre réellement de quel poids pèsent les successions collatérales dans le processus de transmission d'une génération à l'autre nous avons donc cherché à comparer la valeur respective de chacun des deux types de successions mais également à tenir compte de l'âge et du niveau de fortune des défunts sans descendance.

En moyenne les successions en ligne directe s'élèvent à 2998 francs quand les défunts sans descendance laissent 3189 francs. La proximité des deux valeurs est plutôt surprenante, en tous les cas contraire aux hypothèses qui viennent d'être formulées, mais elle est en fait avant tout le résultat de la présence de quelques grosses fortunes, supérieures à 10000 francs, parmi les défunts sans descendance. La valeur médiane des successions en ligne directe est en effet de 942,50 francs alors que le montant de la fortune des défunts sans descendance s'élève seulement à 565 francs. Dans ces conditions la contribution des défunts sans descendance à la transmission intergénérationnelle des patrimoines est moins importante que celle des défunts avec enfants. De ce point de vue le fait même d'avoir une descendance peut apparaître comme un motif suffisant d'accumulation patrimoniale.

La distribution des successions selon la situation familiale du défunt ne permet pas toutefois de conclure que ceux qui n'ont pas d'enfant ont des successions négligeables. En fait, la part des successions inférieures à 100 francs est à peu près équivalente pour les deux populations considérées. La divergence principale, qui explique l'écart important entre la valeur médiane des successions non directes et celle des successions directes, se situe d'une part dans la tranche des successions de 100 à 1000 francs et dans celle des successions de 2000 à 5000 francs. Dans la première tranche se concentrent $54 \%$ des défunts sans enfant contre seulement $41 \%$ des défunts avec une descendance, dans la seconde plus de $20 \%$ des individus ayant des enfants au moment de leur décès contre seulement $10 \%$ des individus sans postérité. En ce sens, le groupe des individus sans descendance n'est pas seulement composé des personnes dont la vie s'achève 
Tableau 2 - Distribution des successions en fonction de leur montant et de la situation familiale de défunt

\begin{tabular}{|l|c|c|c|c|}
\cline { 2 - 5 } \multicolumn{1}{c|}{} & \multicolumn{2}{c|}{ Défunt sans enfant } & \multicolumn{2}{c|}{ Défunt ayant des enfants } \\
\cline { 2 - 5 } \multicolumn{1}{c|}{} & Effectif & pourcentage & Effectif & Pourcentage \\
\hline $0-99 \mathrm{~F}$ & 7 & 8 & 12 & 9,1 \\
\hline $100-999 \mathrm{~F}$ & 47 & 54 & 55 & 41,6 \\
\hline $1000-1999 \mathrm{~F}$ & 14 & 16,1 & 24 & 18,2 \\
\hline $2000-4999 \mathrm{~F}$ & 9 & 10,4 & 27 & 20,4 \\
\hline $5000-9999 \mathrm{~F}$ & 6 & 6,9 & 9 & 6,8 \\
\hline$>10000 \mathrm{~F}$ & 4 & 4,6 & 5 & 3,8 \\
\hline
\end{tabular}

précocement sans qu'elles aient eu le temps de se constituer un patrimoine. Beaucoup au contraire disposent d'une fortune foncière semblable à celle, si l'on peut écrire, du paysan moyen avec enfant. Le célibat définitif important en Bretagne peut donc en partie expliquer cette distribution des successions non directes. Une partie significative des défunts célibataires meurent à un âge suffisamment avancé pour avoir eu le temps de se constituer un patrimoine, sinon important, du moins largement équivalent à celui d'un individu qui s'est marié et a fondé une famille.

\section{Qui sont les défunts sans descendance?}

Cette hypothèse peut cependant être vérifiée de manière relativement simple en comparant la distribution par âges au décès des individus sans enfants et des défunts avec une descendance. Si les registres de mutation par décès sont riches d'informations sur le patrimoine des défunts ils indiquent en revanche rarement leur âge au décès. C'est pourquoi nous avons eu recours aux Tables de successions et absences ${ }^{34}$ qui permettent de retrouver rapidement l'âge de la très grande majorité des défunts.

Tableau 3 - Distribution par âge des défunts selon qu'ils ont ou non une descendance

\begin{tabular}{|l|c|c|}
\hline & Défunts sans enfant & Défunts ayant des enfants \\
\hline$<20$ ans & 13 & 0 \\
\hline $20-29$ & 14 & 1 \\
\hline $30-39$ & 6 & 18 \\
\hline $40-49$ & 6 & 22 \\
\hline $50-59$ & 5 & 15 \\
\hline $60-69$ & 6 & 20 \\
\hline 70 et plus & 13 & 37 \\
\hline Effectif total & 63 & 113 \\
\hline
\end{tabular}

34. Arch. dép. d'Ille-et-Vilaine, 3Q 27450. 
Lâge moyen des défunts sans enfant est de 40,7 ans; il est sensiblement plus bas que celui des individus ayant une descendance (58,5 ans) et semble indiquer que la succession collatérale concerne surtout des défunts morts en pleine jeunesse réduisant ainsi d'autant les effets du célibat définitif sur la transmission intergénérationnelle des patrimoines. Mais ce chiffre recouvre en réalité des situations extrêmement diverses, dont la distribution par tranches d'âge rend mieux compte. Si la moitié des défunts avec une descendance ont plus de 60 ans, c'est le cas d'un tiers des défunts sans postérité. L'autre moitié a entre 30 et 60 ans quand un tiers des individus morts sans enfant se retrouvent dans cette tranche d'âge. Mais la comparaison souffre d'un biais lié aux données elles-mêmes. Par définition, les défunts qui ont une descendance sont quasi inexistants parmi les moins de 20 ans et encore rares avant 30 ans du fait de l'âge tardif au mariage. En revanche, ne pas avoir d'enfant à moins de 30 ans et $a$ fortiori à moins de 20 ans est plus qu'envisageable. En outre, les moins de 30 ans non mariés ne pèsent d'à peu près aucun poids sur la transmission intergénérationnelle des patrimoines; ils n'ont pas eu le temps d'acheter des terres et encore moins de constituer une exploitation ou de faire fortune. Le patrimoine qu'ils lèguent est en fait la plupart du temps constitué de droits successoraux sur le patrimoine d'un de leurs parents prédécédés et revient donc à leurs frères et sœurs survivants qui se redistribuent cette part de droits successoraux. Du point de vue de la transmission des patrimoines, comme du point de vue de la reproduction des générations, ces défunts célibataires de moins de trente ans, à une exception près dans notre échantillon, comptent pour rien ${ }^{35}$.

C'est en effet en prenant en compte seulement les plus de 30 ans que l'on peut observer la place des célibataires à proprement parler mais également celle des couples sans enfant dans la transmission des patrimoines. Parmi les défunts sans enfants dont l'âge est connu les moins de 50 ans sont tous célibataires, à l'exception d'un individu qui meurt marié à 24 ans. Parmi les plus de 50 ans on compte sept personnes mariées, sept veufs et dix célibataires.

Si l'on observe uniquement les plus de 30 ans les deux groupes de défunts avec ou sans descendance offrent le même aspect; la distribution par âge entre les deux groupes d'individus est alors en gros identique. La moitié a entre 30 et 60 ans et l'autre moitié a plus de 60 ans. On mesure également le poids du célibat parmi les 36 défunts sans descendance de plus de 30 ans : 22 ne se sont jamais mariés. En ce sens le célibat apparaît

35. Sur les deux plans strictement de la transmission et de la reproduction des générations, ces célibataires de moins de trente ans ne pèsent pas plus lourd que des enfants morts en bas âge; le registre des successions de Plélan compte d'ailleurs cinq enfants de moins de deux ans. Bien entendu, leur existence jusqu'à l'adolescence ou l'âge adulte n'est pas sans conséquence sur l'économie de leur famille comme charge (bouche à nourrir) ou comme profits (bras) et indirectement sur la politique patrimoniale de leurs parents mais les sources utilisées ici ne permettent pas la prise en compte de ces éléments. 
bien à Plélan comme un élément de régulation du processus de transmission patrimoniale puisque les célibataires forment le groupe majoritaire parmi ceux qui n'ont pas d'enfant mais ont vécu assez longtemps pour se construire un patrimoine à transmettre.

Il ne faudrait donc pas non plus opposer d'une part des individus ayant des enfants dont le patrimoine paraîtrait $a$ priori « constitué " et d'autre part des individus sans descendance dont la fortune serait forcément " inachevée ". En effet passés 30 ans, les deux types d'individus sont également touchés par les risques (mortels) de l'existence. Une moitié de chacun des deux groupes meurt avant l'âge de 60 ans. Les uns et les autres disposent donc en moyenne du même temps pour se constituer un patrimoine, ce qui signifie que l'apport d'un défunt sans descendance à la transmission intergénérationnelle des patrimoines peut être à peu près équivalent à celui d'un individu qui a des enfants. On peut le vérifier en calculant la valeur moyenne des successions des défunts de plus de 30 ans. Les sans descendance ont un patrimoine moyen d'environ 2190 francs $^{36}$. Les individus qui ont des enfants disposent d'une fortune moyenne de 2540 francs ${ }^{37}$ ramenée à 1645 francs si on exclut quatre successions hors norme d'une valeur supérieure à 10000 francs. Nous sommes bien en tout cas dans des ordres de grandeur semblables.

\section{Âge au décès et montant des successions : les effets sur la reproduction familiale}

Que l'on soit marié ou célibataire, avec ou sans descendance, l'âge au décès est une variable indispensable à prendre en compte pour comprendre les comportements patrimoniaux. En effet quelles que soient les intentions des individus, volonté de transmettre un patrimoine le plus important possible ou, au contraire, désir de profiter de leur revenu de leur vivant au point de sacrifier leur capital, la mortalité encore élevée, même à l'âge adulte, au XIX ${ }^{\mathrm{e}}$ siècle bouscule nécessairement ces projets éventuels.

Tableau 4 - Valeur moyenne en francs, des successions selon le type de transmission et l'âge du défunt ${ }^{38}$

\begin{tabular}{|l|c|c|}
\hline & Successions directes & Successions non directes \\
\hline$<30$ ans & & $833(26)$ \\
\hline $30-59$ ans & $1656(51)$ & $1304(17)$ \\
\hline 60 ans et plus & $1653(55)$ & $1862(19)$ \\
\hline
\end{tabular}

36. Moyenne calculée sur 36 cas.

37. Moyenne calculée sur 110 cas.

38. Sont exclues les successions d'une valeur supérieure à 10000 francs. Entre parenthèses est indiqué le nombre de successions ayant servi au calcul de la moyenne. 
À la lumière du tableau 4, l'âge semble avoir un effet réduit sur le montant des patrimoines pour les individus de plus de 30 ans. Au regard des effectifs, quel que soit le type de successions considéré, la valeur moyenne des patrimoines transmis se situe entre 1300 et 1900 francs, ce qui semble invalider l'hypothèse d'un processus d'accumulation au cours de la vie ou celui des effets des accidents de la vie et de la mort prématurée sur le montant des héritages. Seuls les moins de 30 ans ont un patrimoine moyen inférieur à 1000 francs au moment de leur décès, ce qui n'est pas surprenant. Ils sont au début de l'âge adulte, leurs chances d'avoir hérité eux-mêmes sont moindres, leurs acquisitions moins nombreuses faute d'une épargne suffisante. Mais le tableau 4 ne peut que conduire à des conclusions très générales : les tranches d'âge sont trop larges pour permettre une étude fine des effets de l'âge et du cycle de vie sur le montant des successions. La faiblesse des effectifs en ce qui concerne les successions non directes rend ce travail impossible. Il est toutefois bien établi que les célibataires définitifs et les mariés sans enfant disposent à leur décès d'un patrimoine important, comparable en valeur à celui des individus dotés d'une descendance. Pour ces derniers en revanche il est possible d'analyser les effets de l'âge de manière plus approfondie même si les effectifs dans certaines tranches d'âge imposent de rester prudent dans l'interprétation.

Tableau 5 - Valeur moyenne des successions directes selon l'âge du défunt ${ }^{39}$

\begin{tabular}{|l|c|}
\hline Tranche d'âges & Montant moyen de la succession en francs \\
\hline $30-39$ ans & $899(17)$ \\
\hline $40-49$ ans & $2302(19)$ \\
\hline $50-59$ ans & $1697(15)$ \\
\hline $60-69$ ans & $2002(20)$ \\
\hline $70-79$ ans & $1673(25)$ \\
\hline 80 ans et plus & $808(10)$ \\
\hline
\end{tabular}

Les effets du cycle de vie sur le montant des successions apparaissent de manière cependant assez évidentes dans le tableau 5. Les moins de 40 ans qui ont des enfants transmettent des patrimoines de faible valeur, encore inférieure à 900 francs à l'image des célibataires ${ }^{40}$ de moins de 30 ans. Il s'agit d'individus qui sont mariés depuis une dizaine d'années tout au plus. Leur ménage n'a donc pu épargner (ou acheter des biensfonds) que sur un faible laps de temps. En outre, leurs enfants sont encore jeunes et représentent plus souvent une charge qu'un bénéfice en termes de travail et d'épargne. Pour les 40-49 ans la situation est plus favorable, leur temps d'accumulation patrimoniale, par l'achat ou l'épargne, est plus

39. Entre parenthèses sont indiqués les effectifs servant de base au calcul de la valeur moyenne.

40. Très exactement des individus de moins de 30 ans sans enfant. 
long et une partie de leurs enfants au moins est maintenant en âge de participer aux travaux de l'exploitation ou susceptible d'apporter une petit revenu. Les 50-59 ans sont-ils déjà entrés dans un processus de désaccumulation patrimoniale? Ce n'est pas sûr au regard de la valeur moyenne des successions des 60-69 ans qui est plus élevée, même si la faiblesse de nos effectifs explique sans doute en partie cette variation. En fait ce processus de désaccumulation par la vente de biens ou leur donation contre rente en vue de financer ses vieux jours semblent commencer beaucoup plus tardivement. Le mouvement s'amorce à peine chez les 70-79 ans et ne devient significatif que chez les octogénaires.

Au total, les effets de l'âge au décès sur les processus de transmission se font surtout ressentir aux deux extrémités du cycle de vie. Les morts prématurées avant 40 ans mettent les enfants, souvent mineurs, voire en bas âge, dans une situation délicate en termes d'apport par l'héritage. Il en va de même pour ceux dont les parents meurent après 80 ans. En revanche, pour ceux dont les parents meurent entre 40 et 80 ans, le montant des héritages reçus est beaucoup plus substantiel. D'une certaine manière, tous les accidents de la vie (en l'occurrence les morts " prématurées " des parents, même au-delà de l'âge de 40 ou 50 ans) n'ont pas tous d'effets si lourds sur les transferts intergénérationnels de patrimoine, sauf pour ceux qui perdent un père ou une mère âgé de moins de 40 ans. Pour ceux dont les parents vivent âgés (au-delà de 70 ans) et qui doivent attendre longtemps leur héritage, les espoirs en ce domaine sont également plus réduits. Une vie " sans accident ", parce qu'elle dure " trop " longtemps en quelque sorte, grignote alors l'héritage que les enfants peuvent escompter. Les vieux parents sont en effet souvent conduits à consommer leur capital $^{41}$, c'est-à-dire à vendre une partie des biens qu'ils ne sont plus capables d'exploiter pour vivre au quotidien au détriment de l'héritage qu'ils laisseront à leurs enfants.

Tel qu'il apparaît ici, le rôle de l'âge sur la fortune des défunts à Plélan invite finalement à reposer la question du lien entre patrimoine et descendance. Car si, comme nous l'avons montré précédemment, le risque d'éparpillement des patrimoines d'une génération à l'autre considérée dans leur ensemble est considérablement réduit dans le canton de Plélan par la proportion importante de célibataires définitifs, les individus qui ont une descendance, et qui sont donc les premiers concernés par le problème de la transmission, ne semblent pas prendre forcément en compte cette donnée dans leurs comportements patrimoniaux. En effet les données disponibles, même si elles sont parfois rendues fragiles par la faiblesse des effectifs, n'indiquent pas de manière évidente de processus d'accumulation

41. On peut faire ici d'ailleurs le parallèle avec les problèmes actuels de financement du quatrième âge. L'une des solutions envisagées, notamment par certains conseils généraux en charge d'une partie de son financement, est de prélever une partie de l'héritage au décès de la personne âgée pour compenser le coût engendré par cette longévité pour la collectivité, et ce donc aux dépens des héritiers. 
continu en vue de transmettre, ce que les économistes ${ }^{42}$ appelleraient un " comportement altruiste ". Le patrimoine est maintenu intact une fois la vieillesse venue (jusqu'à 70 ans au moins) mais semble abandonné par les plus vieux (notamment après 80 ans).

\section{Existe-t-il un lien entre limitation de la descendance et accumulation patrimoniale?}

Cette déperdition de patrimoine pourrait cependant s'expliquer paradoxalement par une volonté de transmettre. Les très vieux feraient des donations à leurs enfants et anticiperaient ainsi leur succession, ce qui expliquerait le faible montant des héritages des défunts de plus de 80 ans et laisserait supposer une volonté d'assurer un bon transfert intergénérationnel du patrimoine familial. La consultation des archives notariales de la région ne laisse pourtant pas apparaître l'anticipation de succession par donation comme une pratique courante au milieu du XIX ${ }^{\mathrm{e}}$ siècle dans le canton de Plélan. En tous les cas une telle volonté n'est pas corrélée à un comportement démographique approprié. Les Bretons ne pratiquent guère dans leur ensemble, on l'a vu, la limitation des naissances dans le canton. Peut-on néanmoins distinguer chez ceux dont la descendance est réduite une volonté de transmettre dans les meilleures conditions leur patrimoine et/ou leur exploitation?

Tableau 6 - Valeur moyenne des successions en fonction du nombre d'enfants du défunt ${ }^{43}$

\begin{tabular}{|c|c|}
\hline Nombre d'enfants & Valeur moyenne de la succession \\
\hline 1 & $878(17)$ \\
\hline 2 & $1296(24)$ \\
\hline 3 & $1821(21)$ \\
\hline 4 & $1706(26)$ \\
\hline 5 & $1601(15)$ \\
\hline 6 & $2595(15)$ \\
\hline 7 et plus & $2147(10)$ \\
\hline
\end{tabular}

Au regard du tableau 6 une descendance limitée (moins de deux enfants au moment du décès) ne s'accompagne pas d'une concentration accrue de patrimoine puisque la valeur moyenne des successions des individus concernés s'établit autour de 1000 francs. Les défunts qui ont trois à cinq enfants ont un patrimoine d'une valeur sensiblement plus importante située

42. ARrondel, Luc et Grange, Cyril, "Successions et héritiers dans la société rurale du XIX ${ }^{\mathrm{e}}$ siècle : l'exemple des familles TRA de Loire-inférieure ", Annales de démographie historique, 2004-1, p. 53-77.

43. Successions supérieures à 10000 francs exclues. Entre parenthèses est indiqué le nombre de succession servant au calcul de la moyenne. 
entre 1600 et 1800 francs. Enfin les individus qui laissent une famille très nombreuse (six enfants et plus) ont un patrimoine encore plus important, supérieur à 2100 francs. L'idée selon laquelle le petit paysan propriétaire triomphant du XIX ${ }^{\mathrm{e}}$ siècle ${ }^{44}$ chercherait à limiter sa descendance afin d'assurer à la fois la pérennité de l'exploitation et l'avenir de ses enfants ne se vérifie donc pas à Plélan. À Vernon dans les années 1810, l'analyse des successions conduisait aux mêmes conclusions, à Bayeux et à Livarot également ${ }^{45}$. En revanche, l'enquête menée par L. Arrondel et C. Grange sur les successions en milieu rural à partir des familles TRA de Loire-inférieure arrivait à des conclusions inverses puisque les auteurs notent "l'influence positive d'une descendance limitée (2 enfants) sur le montant transmis, révélant l'existence d'un motif de transmission au XIX ${ }^{\mathrm{e}}$ siècle ${ }^{46}$ ". Si les données de Plélan indiquent clairement qu'il n'y a pas de corrélation, sinon négative, entre montant des successions et limitation de la descendance, il est difficile d'en faire un cas généralisable. Pour autant cette situation ne peut être considérée seulement comme la conséquence du maintien d'une fécondité élevée en Bretagne au XIX siècle. La Loire-inférieure sans être sur le même modèle démographique que le reste de la Bretagne a encore $\mathrm{au} \mathrm{XIX}^{\mathrm{e}}$ siècle une fécondité bien supérieure à celle des départements normands ${ }^{47}$. Elle se signale elle aussi au milieu du XIX ${ }^{\mathrm{e}}$ siècle par un taux de célibat définitif parmi les plus élevés de France ${ }^{48}$. En outre, même les Normands à Vernon semblent en effet avoir intégré les principes de la limitation des naissances sans que cela se traduise par des comportements malthusiens sur le plan patrimonial, c'est-à-dire une corrélation entre le nombre d'enfants et le montant des successions.

Si le fait d'avoir une descendance nombreuse favorise la constitution d'un patrimoine foncier plus important, pourtant destiné à être morcelé à la génération suivante, on doit alors se demander jusqu'à quel point le motif de transmission permet d'expliquer les comportements patrimoniaux ${ }^{49}$. Les enfants sont à la fois une charge et une richesse du point de vue de l'économie du ménage. Selon les étapes de son cycle de vie, avoir une descendance nombreuse peut ainsi favoriser l'augmentation du patrimoine foncier. Si les enfants sont en âge de travailler, ils peuvent à la fois permettre de travailler une surface plus importante mais également apporter des revenus supplémentaires au ménage et permettre ainsi de dégager une épargne plus importante en vue d'une acquisition foncière. De plus, un nombre

44. MAYAUD, Jean-Luc, La Petite Exploitation rurale triomphante, Paris, Belin, 1999.

45. BÉAUR, Gérard, "Trop de stratégie? ", op. cit., p. 48-49.

46. ARRondel, Luc, et GRANGe, Cyril, "Successions et héritiers... ", art. cit., p. 67.

47. La Loire-inférieure a un indice de Coale $I_{f}$ de 0,369 en 1806 (p. 198) et de 0,299 en 1856 (Bonneul, Noël, Transformation of the French..., op. cit., p. 201).

48. DuPÂQUIER, Jacques (dir.), Histoire de la population..., op. cit., p. 385.

49. On pourrait raisonner de manière inverse en suggérant que la possession d'un patrimoine foncier important permet d'entretenir une descendance nombreuse. Mais ce raisonnement bute sur la faible valeur de la très grande majorité des patrimoines dans la région, qui ne suffit pas à l'entretien d'une famille sans l'apport de revenus annexes, notamment ceux issus du marché du travail. 
important d'enfants peut permettre aux parents âgés de conserver plus longtemps leur exploitation. La présence d'enfants célibataires au foyer ou d'enfants installés à proximité peut aider les vieux parents à conserver leurs biens-fonds au lieu qu'ils soient contraints de les vendre pour financer leurs consommations quand leurs forces déclinent. Bien entendu ce raisonnement ne peut s'appliquer à tous les types de successions et à tous les types d'individus. La corrélation entre patrimoine, importance de la descendance et étapes du cycle de vie n'a de sens que pour des individus dont le revenu dépend étroitement de leur capital foncier et de leur travail. En clair cette hypothèse n'a de sens que pour des individus qui vivent essentiellement en travaillant eux-mêmes leurs terres. Les très grands propriétaires fonciers, qui vivent de la rente foncière et non du travail de la terre, ne sont pas soumis de la même manière et avec la même intensité aux contraintes du cycle de vie familial ${ }^{50}$. Arrivés à des âges avancés leurs rentes continuent d'assurer le financement de leur consommation sans qu'ils aient besoin d'entamer leur capital foncier au contraire du petit paysan ou du moyen propriétaire dont les revenus dépendent fortement de sa capacité de travail propre et de celle des membres de son ménage ou de sa famille proche. De ce point de vue, le motif de transmission et les théories économiques malthusiennes du XIX ${ }^{\mathrm{e}}$ siècle ne peuvent s'appliquer de manière univoque à l'ensemble de la société. Sans doute existe-t-il des effets de seuil de fortune en deçà desquels les contraintes du cycle de vie et en particulier de la vieillesse et de son financement imposent d'autres logiques de comportement.

Pour aller plus avant dans l'examen des liens entre succession et descendance et comprendre quelle place occupe le motif de transmission dans le comportement patrimonial des paysans, il faudrait sans doute disposer de données beaucoup plus importantes. Il faudrait notamment être en mesure d'étudier les effets propres de la taille de la descendance sur le montant des successions par une analyse par tranche d'âge. Avoir six enfants n'a pas le même sens pour un individu qui meurt à 75 ans ou pour un défunt mort " prématurément " à 40 ans. Dans le premier cas il s'agit d'une descendance définitive tandis que dans le second la mortalité au cours de l'enfance peut encore faire évoluer significativement la taille de sa descendance. Le premier a pu développer dans sa totalité sa "stratégie " patrimoniale, si il en a une, tandis que le second n'en a pas eu le temps.

La transmission du patrimoine foncier apparaît a priori comme l'élément central du processus de reproduction sociale dans une économie encore largement agricole. En Bretagne, le maintien d'une forte fécondité durant tout le $\mathrm{xIX}^{\mathrm{e}}$ siècle se traduit par une importante descendance des défunts et devrait donc accroître un peu plus le problème du morcellement

50. Sur les très grands propriétaires et le problème du morcellement des domaines au XIX ${ }^{\mathrm{e}}$ siècle, voir THÉBAULT, Vincent, "La faute des pères. Fragmentation patrimoniale et représentation du déclin social ", Ruralia, n 10 /11-2002, p. 147-179. 
des propriétés et les difficultés d'installation des enfants. Pourtant, considéré de manière globale, c'est-à-dire au niveau des transferts intergénérationnels au sein du canton, le problème est moins important qu'on peut le penser a priori. La démographie règle en grande partie la question du morcellement, même dans le canton breton de Plélan. Certes la réponse est différente de celle d'autres régions mais elle aboutit à un résultat similaire. Compensant une fécondité encore importante par un taux de célibat plus fort, la Bretagne parvient à rendre le transfert des patrimoines et des exploitations moins fréquemment compliqué. Plus de la moitié des successions se déroulent dans de bonnes conditions de ce point de vue puisque plus la moitié des défunts n'ont pas d'enfant ou n'en ont pas plus de deux.

Néanmoins, bien que les situations y soient presque équivalentes au niveau global, la transmission des patrimoines semble tout de même plus fréquemment problématique en Bretagne qu'en Normandie au niveau des familles. Les familles nombreuses y sont en effet plus fréquentes. Toutefois, le nombre important de successions collatérales vient atténuer les effets de la taille des fratries d'héritiers. L'oncle ou la tante sans descendance vient grossir l'héritage des neveux et nièces ${ }^{51}$. Cet apport est d'autant moins négligeable que le montant moyen des successions collatérales provenant de véritables célibataires (de plus de 30 ans) est tout à fait comparable à celui d'un individu marié avec enfant. Dans une région où le recours au testament reste limité et où les défunts sans descendance laissent le plus souvent le Code civil désigner leurs héritiers, chaque neveu ou nièce peut intégrer à ses espoirs d'héritage une part de la succession de son oncle ou de sa tante célibataire. Sur les 87 défunts sans descendant, 22 ont, d'après le registre de mutation de 1856, rédigé un testament ou effectué une donation au préalable. C'est donc bien seulement dans les familles nombreuses, qui comptent quatre enfants ou plus et qui représentent tout de même quatre ménages sur dix, que la transmission de l'exploitation ou d'un patrimoine suffisant pour asseoir sa reproduction sociale est la plus délicate.

Ces difficultés ouvrent-elles pour autant, chez certains individus, la voie à la mise en œuvre de stratégies pour limiter le morcellement du patrimoine à la génération suivante? Bien entendu la seule analyse des successions ne permet pas de rendre compte de toutes les actions éventuelles entreprises dans ce but. Il est néanmoins clair que dans le cas du canton de Plélan la limitation de la descendance n'est pas l'instrument d'une politique de préservation du patrimoine au profit de la génération suivante. La limitation de la descendance ne s'accompagne pas d'une accumulation accrue de patrimoine mais plutôt d'une moindre fortune. En ce sens le schéma malthusien qui, au XIX ${ }^{\mathrm{e}}$ siècle, s'applique aux élites et permet de faire le lien entre des pratiques contraceptives, une limitation des naissances et une volonté de transmission du patrimoine, ne peut s'étendre aux populations rurales de la région de Plélan. L'émergence de la figure du petit propriétaire

51. TREVISI, Marion, Au cœur de la parenté. Oncles et tantes dans la France des Lumières, Paris, PUPS, 2008, « Transmettre », p. 263-312. 
attaché à la conservation et à la transmission de son bien ne se traduit pas par un comportement démographique spécifique ni par la mise en œuvre d'une politique de préservation du patrimoine au bénéfice de la génération suivante. On pourrait au contraire affirmer, et pas seulement par goût du paradoxe, qu'une descendance nombreuse préserve le patrimoine des parents aux dépens des enfants; les premiers en s'appuyant sur les seconds, leur travail et une partie de leurs revenus, peuvent accroître leur capacité d'épargne et arrondir leur patrimoine foncier mais au détriment de leurs enfants qui reçoivent des portions d'héritage plus réduites que les enfants uniques ou que les petites fratries.

S'il est certain que la transmission intergénérationnelle des exploitations pose le problème de leur morcellement, dans une grosse minorité de cas le cœur du problème de la reproduction sociale se situe également ailleurs au milieu du XIXe siècle dans ces campagnes bretonnes de la région de Plélan : La vie " accidentée " pèse d'un poids autrement plus important sur le destin des enfants. La mort prématurée (surtout avant 40 ans) d'un ou des parents complique sans doute davantage les conditions de la reproduction sociale et familiale des héritiers. Alors qu'ils sont encore mineurs, ou en tout cas encore très jeunes au moment de l'héritage, la mort de leur parent intervient pour eux avant qu'ils n'aient eu le temps de s'installer dans la vie conjugale et dans le monde du travail. Mais surtout leur part d'héritage est réduite par le fait que leurs parents ne disposent pas d'un patrimoine aussi important qu'ils pouvaient l'espérer s'ils étaient morts plus tard. Enfin, la vieillesse des parents vient, mais dans une moindre mesure, réduire le bénéfice escompté par les héritiers. Passés 70 ans et surtout après 80 ans, beaucoup doivent entamer leur capital foncier pour financer leurs consommations; ils réduisent alors la valeur de leur succession. Mais les effets pour les enfants sont moins douloureux que dans le cas des morts prématurées car ces décès interviennent à des moments plus favorables du cycle de vie des enfants qui sont alors adultes et déjà installés.

Poser la question de la reproduction familiale au milieu du XIX ${ }^{\mathrm{e}}$ siècle en termes de maintien de l'exploitation sur plusieurs générations n'est donc pas forcément la manière, sinon la plus appropriée, du moins exclusive, de rendre compte des difficultés que rencontrent les individus pour trouver des moyens d'existence dans une région à l'activité agricole traditionnelle encore dominante. D'une part, la démographie continue d'agir comme un régulateur du système de transmission même dans une région marquée encore au XIXe siècle par une fécondité élevée. D'autre part, entre ceux qui voient leurs parents mourir trop tôt pour laisser un héritage important et ceux qui les voient vivre vieux et ont déjà entamé leur vie professionnelle et leur propre parcours patrimonial au moment du décès de leurs parents, l'héritage foncier proprement dit semble n'intervenir finalement de manière décisive dans le processus de reproduction sociale et familiale que pour une faible part de la population. Situation paradoxale pour une société rurale où la rente foncière reste le fondement des fortunes. 


\section{RÉSUMÉ}

Dans une économie agricole, la transmission du patrimoine foncier semble a priori être un élément essentiel de la reproduction sociale et familiale. En utilisant les registres de mutation par décès de Plélan-le-Grand (Ille-et-Vilaine) de 1856 qui indiquent le montant des fortunes des défunts et le nom de leurs héritiers, cet article essaie de mesurer précisément le rôle de l'héritage sur la reproduction sociale dans une région qui a encore une forte fécondité au XIX ${ }^{e}$ siècle. L'analyse des données bretonnes (que nous comparons aux données normandes) révèle que le problème de la transmission doit être relativisé car beaucoup d'individus restent célibataires, ce qui diminue l'impact de la forte fécondité des Bretonnes. En outre, l'impact de l'héritage sur la reproduction sociale est aussi atténué en fonction de l'âge des parents quand il meurt : les pères et les mères qui meurent jeunes ou très vieux (plus de 70 ans) ont en effet des fortunes moins importantes. Enfin l'article pose la question des stratégies de transmission chez les individus qui réduisent volontairement leur descendance.

\section{ABSTRACT}

In an agricultural economy, the transfer of the land seems a priori to be an essential element of social and family reproduction. Using the records of "mutation par décès" of Plélan-le-Grand (Ille-et-Vilaine, 1856) that reflect the fortunes of the deceased and the names of their heirs, this article tries to measure precisely the role of inheritance on social reproduction in a region that still has a high fertility in the nineteenth century. Data analysis (which we compare to data in Normandy) indicates that the problem of transmission has to be relativized because many people remain single, which reduces the impact of high fertility in Bretagne. In addition, the impact of inheritance on social reproduction is also reduced with age of parents when he died: Fathers and mothers who die young or very old (over 70 years) have indeed smaller fortunes. Finally in this article it is discussed the question of transmission strategies for individuals who voluntarily reduce the number of their children. 